\title{
Clinical features and predictors of masked uncon- trolled hypertension from the Korean Ambulatory Blood Pressure Monitoring Registry
}

Hyun-Jin Kim ${ }^{1, *}$, Jeong-Hun Shin ${ }^{1, *}$, Yonggu Lee ${ }^{1}$, Ju Han Kim², Sun Ho Hwang ${ }^{3}$, Woo Shik Kim4, Sungha Park ${ }^{5}$, Sang Jae Rhee ${ }^{6}$, Eun Mi Lee ${ }^{7}$, Sang Hyun Ihm ${ }^{8}$, Wook Bum Pyun ${ }^{9}$, and Jinho Shin ${ }^{1}$

Division of Cardiology, Department of Internal Medicine, ${ }^{1}$ Hanyang University College of Medicine, Seoul; ${ }^{2}$ Chonnam National University Hospital, Gwangju; ${ }^{3}$ Gwangju Veterans Hospital, Gwangju; ${ }^{4}$ Kyung Hee University Medical Center, Seoul; ${ }^{5}$ Severance Cardiovascular Hospital, Yonsei University College of Medicine, Seoul; 'Wonkwang University Hospital, Iksan; ${ }^{7}$ Wonkwang University Sanbon Hospital, Gunpo; ${ }^{8}$ College of Medicine, The Catholic University of Korea, Seoul; ${ }^{9}$ Ewha Womans University Seoul Hospital, Seoul, Korea

Received: December 7, 2020 Revised : February 8, 2021 Accepted: March 22, 2021

\section{Correspondence to}

Jinho Shin, M.D.

Division of Cardiology,

Department of Internal

Medicine, Hanyang University

Medical Center, 222-1

Wangsimni-ro, Seongdong-gu,

Seoul 04763 , Korea

Tel: +82-2-2290-8308

Fax: +82-2-2299-0278

E-mail:jhs2003@hanyang.ac.kr https://orcid.org/0000-00016706-6504

*'These authors contributed equally to this work.
Background/Aims: The clinical characteristics of patients with masked uncontrolled hypertension (MUCH) have been poorly defined, and few studies have investigated the clinical predictors of MUCH. We investigated the demographic, clinical, and blood pressure (BP) characteristics of patients with MUCH and proposed a prediction model for MUCH in patients with hypertension.

Methods: We analyzed 1,986 subjects who were enrolled in the Korean Ambulatory Blood Pressure Monitoring (Kor-ABP) Registry and taking antihypertensive drugs, and classified them into the controlled hypertension $(\mathrm{n}=465)$ and MUCH (n = 389) groups. $\mathrm{MUCH}$ was defined as the presence of a 24-hour ambulatory mean systolic $\mathrm{BP} \geq 130 \mathrm{mmHg}$ and/or diastolic $\mathrm{BP} \geq 80 \mathrm{mmHg}$ in patients treated with antihypertensive drugs, having normal office BP.

Results: Patients in the MUCH group had significantly worse metabolic profiles and higher office BP, and took significantly fewer antihypertensive drugs compared to those in the controlled hypertension group. Multivariate logistic regression analyses identified high office systolic BP and diastolic BP, prior stroke, dyslipidemia, left ventricular hypertrophy ( $\mathrm{LVH}, \geq 116 \mathrm{~g} / \mathrm{m}^{2}$ for men, and $\geq 96 \mathrm{~g} / \mathrm{m}^{2}$ for women), high heart rate ( $\geq 75$ beats/min), and single antihypertensive drug use as independent predictors of MUCH. A prediction model using these predictors showed a high diagnostic accuracy (C-index of o.839) and goodness-of-fit for the presence of MUCH.

Conclusions: $\mathrm{MUCH}$ is associated with a high-normal increase in office BP and underuse of antihypertensive drugs, as well as dyslipidemia, prior stroke, and LVH, which could underscore achieving optimal BP control. The proposed model accurately predicts MUCH in patients with controlled office BP.

Keywords: Masked uncontrolled hypertension; Blood pressure monitoring, ambulatory; Blood pressure 


\section{INTRODUCTION}

Masked hypertension ( $\mathrm{MH}$ ) is defined as the presence of normal office blood pressure (BP) but high out-ofoffice BP, as determined by 24-hour ambulatory blood pressure monitoring (ABPM) or home BP monitoring [1]. As $\mathrm{MH}$ could also refer to those individuals not taking antihypertensive drugs, high out-of-office BP with normal office BP in patients on antihypertensive therapies, which was first described 10 years ago, was later renamed as masked uncontrolled hypertension (MUCH) [2]. $\mathrm{MH}$ is known to increase the risk of cardiovascular diseases as it is often undetected and untreated [3]. Previous studies on $\mathrm{MH}$ included patients with $\mathrm{MUCH}$ and did not distinguish cardiovascular risks between patients receiving antihypertensive therapies and those not receiving antihypertensive therapies [4-6]. Other studies previously reported significantly higher cardiovascular risk in patients with MUCH than in those with controlled hypertension [7-9]. The prognostic value of MUCH is not yet clear, but it is inseparable from $\mathrm{MH}$ in terms of increased cardiovascular risk. In outpatient department settings, where office BP remains the main BP assessment tool, no effective indicator has been widely used to detect MUCH, and therefore, it is difficult to suspect in daily practice. It is desirable to recognize clinical features strongly related to the presence of $\mathrm{MUCH}$ and to utilize these features as an effective screening tool for MUCH that can be easily availed in the outpatient setting. In this study, we investigated the clinical characteristics of MUCH and identified significant predictors for the presence of $\mathrm{MUCH}$, using which we developed a prediction model for MUCH in patients who were treated with antihypertensive drugs.

\section{METHODS}

\section{Study design and setting}

Cross-sectional study data were obtained from the nationwide Korean ABPM (Kor-ABP) Registry for Evaluation of the Prognostic Threshold in Hypertension, organized by the Korean Society of Hypertension. This prospective multicenter cohort included patients who underwent 24-hour ABPM to evaluate high BP. The Kor$\mathrm{ABP}$ registry involved outpatient clinics in 27 tertiary and secondary hospitals between August 2009 and December 2016 [10]. Treatment and drug administration were performed at the discretion of physicians for patients with hypertension, and all investigators were cardiologists who were experts on hypertension and clinical cardiology. Among the initially enrolled 5,965 patients who had undergone 24-hour ABPM, 5,404 patients had valid 24-hour ABPM data. Of these, 1,986 patients were treated with antihypertensive drugs. A total of 1,132 patients with elevated office BP (systolic blood pressure $[\mathrm{SBP}] \geq$ $140 \mathrm{mmHg}$ and/or diastolic blood pressure $[\mathrm{DBP}] \geq 90$ $\mathrm{mmHg}$ ) were excluded. Therefore, 854 patients treated with antihypertensive drugs with normal office BP (SBP $<140 \mathrm{mmHg}$ and/or DBP < $90 \mathrm{mmHg}$ ) were included in the final analysis, and we classified the patients into groups according to BP characteristics obtained from 24-hour ABPM as the MUCH and controlled hypertension groups (Fig. 1).

The study protocol complied with the Declaration of Helsinki, and was reviewed and approved by the Institutional Review Board of each center, including Hanyang

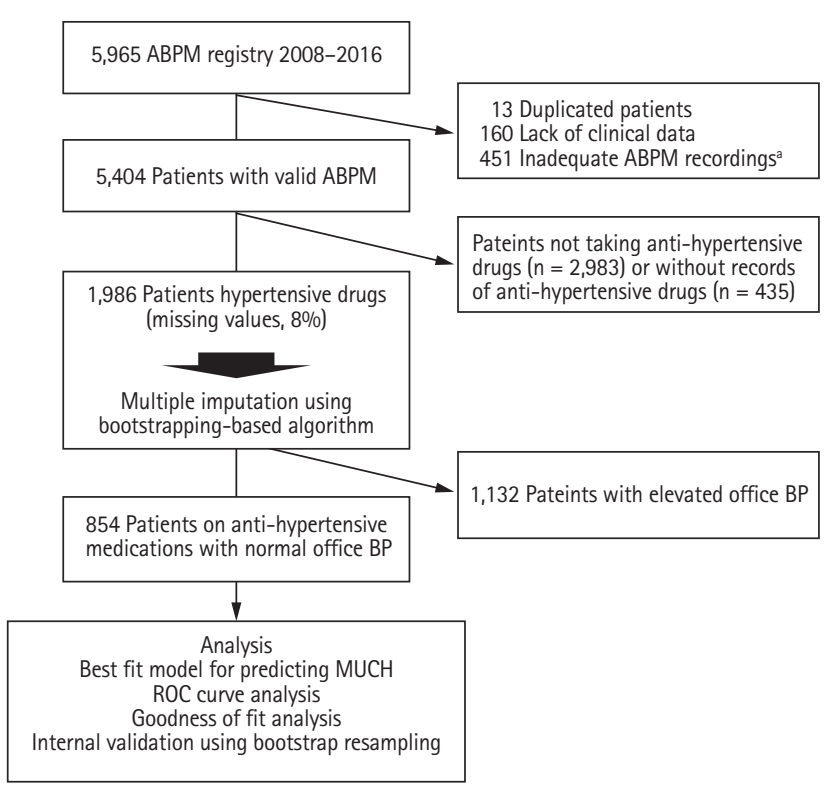

Figure 1. Study population. ABPM, ambulatory blood pressure monitoring; BP, blood pressure; MUCH, masked uncontrolled hypertension; ROC, receiver operating characteristic. ${ }^{a}$ At least 20 recordings during wakefulness and seven recordings during sleep, after the exclusion of unmeasured recordings and recordings with extreme blood pressures (systolic BP $\geq 350$ or $<60 \mathrm{mmHg}$; diastolic $\mathrm{BP} \geq 250$ or $<30$ $\mathrm{mmHg}$ ). 
University Seoul Hospital (HYUH IRB No. 2009-R-12). Written informed consent was obtained from all participants.

\section{Data collection}

Data were collected using a web-based electronic data capture system that included electronic case report forms from the Kor-ABP registry database. The following demographic and clinical characteristics were extracted: sex, age, body mass index (BMI), waist circumference (WC), extent of exercise, smoking status, alcohol intake, and traditional cardiovascular risk factors, including a history of hypertension, diabetes, dyslipidemia, stroke, myocardial infarction (MI), heart failure, and malignancy. Family history of hypertension, diabetes, and cardiovascular death was also obtained, along with data on antihypertensive drugs.

The following laboratory data were extracted: hemoglobin, triglyceride, total cholesterol, high-density lipoprotein cholesterol, low-density lipoprotein cholesterol (LDL-C), estimated glomerular filtration rate $(\mathrm{mL} /$ $\min / 1.73 \mathrm{~m}^{2}$ ), hemoglobin Arc, and fasting glucose. Left ventricular ejection fraction and left ventricular mass index (LVMI) were extracted from M-mode echocardiographic estimation data. Left ventricular hypertrophy $(\mathrm{LVH})$ was defined as LVMI $\geq 116 \mathrm{~g} / \mathrm{m}^{2}$ for men and $\geq 96$ $\mathrm{g} / \mathrm{m}^{2}$ for women.

\section{ABPM data and definition of groups}

A raw ABPM dataset was considered valid only if it contained at least 20 valid recordings from while awake and seven valid recordings from during sleep, after excluding unmeasured recordings and recordings with extreme $\mathrm{BP}(\mathrm{SBP} \geq 350$ or $<60 \mathrm{mmHg}$; $\mathrm{DBP} \geq 250$ or $<30 \mathrm{mmHg}$ ) [11]. Office BP was measured using a UA767 monitor (A\&D Co., Ltd., Tokyo, Japan), which had passed the European Hypertension Society protocol at all institutions. Office BP was determined as the mean BP of two measurements taken at 1 minute intervals with a 5-minute rest before the first measurement [12].

MUCH was defined as the presence of 24 -hour ambulatory mean SBP of $\geq 130 \mathrm{mmHg}$ and/or DBP of $\geq 80$ $\mathrm{mmHg}$ in patients treated with antihypertensive drugs with normal office $\mathrm{BP}$ (SBP of $<140 \mathrm{mmHg}$ and/or DBP of $<90 \mathrm{mmHg}$ ), according to the international guideline criteria for $\mathrm{MH}[1,13]$. Controlled hypertension was de- fined as 24-hour ambulatory mean SBP/DBP of < 130/80 $\mathrm{mmHg}$ and office SBP/DBP of $<140 / 90 \mathrm{mmHg}$ in patients receiving an antihypertensive drug.

\section{Statistical analyses}

The study population with normal office BP was divided into two groups according to the presence of high 24-hour ABPM (controlled hypertension or MUCH). Continuous variables such as age, BMI, and WC, and categorical variables such as sex and number of antihypertensive drugs in the two groups were compared using Student's $t$ test and chi-square test, respectively. Fisher's exact test was used for non-parametric analyses.

Because $8 \%$ of the data in the original dataset was missing (Supplementary Fig. 1), we performed multiple imputations using a bootstrap expectation-maximization algorithm. Five possible imputed datasets were created, and the average value of the five imputed values was adopted as the missing values for the continuous variables. The most frequent value of the five imputed values was adopted for the categorical variables.

Univariate logistic regression analyses were used to evaluate the association between clinical factors and MUCH. To recognize the non-linearity of the associations with continuous variables (e.g., age, LVMI, heart rate [HR], BMI, and WC) and determine the optimal cut-off points for the variables, restrictive cubic spline fits were used in the univariate logistic regression analyses. A multivariate logistic regression model was developed to identify significant predictors of $\mathrm{MUCH}$ in patients with controlled office BP. All available potential confounders including age, sex, BMI, WC, exercise < 3 times/week, current alcohol intake or smoking, diabetes and dyslipidemia, history of stroke, MI or malignancy, family history of hypertension, diabetes or cardiovascular death, type and number of antihypertensive drugs, office SBP, office DBP, HR, and LDL-C levels were entered into the multivariate model as covariates to avoid prejudice. The multivariate model was reduced using a backward variable selection process to minimize overfitting of biases and to find the best-fit model (cut-off criteria, $p<0.05$ ).

A prediction scoring system for MUCH was produced using the coefficients of significant predictors from the best-fit model. The largest among the coefficients of predictors was set as the integer 10, the maximum 
point in the nomogram, and the other coefficients were rescaled relative to the maximum point. The total score was calculated as the sum of the points assigned to all significant predictors included in the best-fit model, as previously described [14]. Receiver operating characteristic (ROC) curve analysis was conducted using the linear predictors derived from the best-fit model to evaluate the performance of the prediction scoring system for MUCH. Sensitivity, specificity, positive predictive value (PPV), and negative predictive value (NPV) were calculated at an optimal cut-off point derived from Youden's J-index. The goodness of fit between the predicted probability and the observed incidence of $\mathrm{MUCH}$ was evaluated using the Hosmer-Lemeshow test. The observed incidence of MUCH was calculated at every score interval of 1 for the Hosmer-Lemershow test.

We conducted internal validation and calibration of the best-fit model using bootstrap resampling [15]. We validated the full logistic regression model with all covariates using 1,000 bootstrap resamples. In each bootstrap dataset, the full model with all covariates was reduced to a bootstrap model using a backward selection process, and optimism was estimated using the C-indexes of the best-fit and bootstrap models [16]. The number of absolute errors between the predicted probability and the actual probability of MUCH in the best-fit model was also estimated through a calibration procedure using the same 1000 bootstrap re-samples.

All statistical analyses were performed using the statistical software R3.6.1, Rstudio1.2.1, and its packages including "descry," "rms," "Amelia," "tableone," "coin," "ROCR," and "pROC." Statistical significance was set at $p<0.05$.

\section{RESULTS}

\section{Baseline characteristics}

Among the 1,986 patients treated with antihypertensive drugs, 854 had normal office BP. Among the patients with apparently normal office BP, $45.6 \%$ were classified into the MUCH group $(\mathrm{n}=389)$ and $54.4 \%$ were classified into the controlled hypertension group $(n=465)$. Comparisons between baseline characteristics of the controlled hypertension and MUCH groups are presented in Table 1. Patients in the MUCH group were more frequently alcohol drinkers and presented with poorer metabolic profiles, including higher obesity indexes and serum cholesterol levels, compared with those in the controlled hypertension group. Office SBP and DBP were higher, and a history of stroke was slightly more common in the MUCH group than in the controlled hypertension group. In addition, the MUCH group took significantly fewer antihypertensive drugs than the controlled hypertension group, and the number of patients taking a single antihypertensive drug was significantly higher in the MUCH group than in the controlled hypertension group.

\section{Prevalence of $\mathrm{MUCH}$ according to BP}

The higher the office SBP and DBP, the higher the prevalence of MUCH (Fig. 2). However, when the office SBP was $\geq 130 \mathrm{mmHg}$, the prevalence of $\mathrm{MUCH}$ was similarly high at all DBP levels. The prevalence of $\mathrm{MUCH}$ did not differ according to the number of antihypertensive drugs; however, when the SBP was $\geq 130 \mathrm{mmHg}$, the prevalence of MUCH decreased with the increasing number of antihypertensive drugs.

\section{Predictors for $\mathrm{MUCH}$}

The prevalence of $\mathrm{MUCH}$ and odds ratios (OR) with 95\% confidence intervals (CI) for the presence of $\mathrm{MUCH}$ from univariate logistic regression analyses are presented according to various clinical factors in Table 2. An office SBP of 120 to $129 \mathrm{mmHg}$ or 130 to $139 \mathrm{mmHg}$, office DBP of 80 to $84 \mathrm{mmHg}$ or 85 to $89 \mathrm{mmHg}$, single antihypertensive drugs, history of stroke, $\mathrm{HR} \geq 75$ beats/ $\min , \mathrm{BMI} \geq 25 \mathrm{~kg} / \mathrm{m}^{2}$, central obesity, LVH, male sex, and current alcohol intake were associated with the presence of MUCH (Table 2). In the multivariate logistic regression analysis, the best-fit model obtained after the backward variable selection process showed that office SBP, office DBP, stroke, dyslipidemia, LVH, HR $\geq 75$ beats/ minute, and the number of antihypertensive drugs used were significantly associated with the presence of MUCH (Table 3). In particular, the high-normal office SBP (130 to $139 \mathrm{mmHg}$ ) and DBP ( 85 to $89 \mathrm{mmHg}$ ) were most strongly associated with the presence of MUCH. The use of a smaller number of antihypertensive drugs was also associated with the presence of $\mathrm{MUCH}$, when compared to the use of three drugs or more.

We also analyzed the association between the types 
Table 1. Comparison of the baseline characteristics between the controlled HTN and MUCH groups

\begin{tabular}{|c|c|c|c|}
\hline Characteristic & $\begin{array}{l}\text { Controlled HTN } \\
(\mathrm{n}=465)\end{array}$ & $\begin{array}{l}\text { Masked uncontrolled HTN } \\
\qquad(\mathrm{n}=389)\end{array}$ & $p$ value \\
\hline Male sex & $241(51.8)$ & $236(60.7)$ & 0.012 \\
\hline Age, yr & $61.1 \pm 11.6$ & $60.2 \pm 12.9$ & 0.268 \\
\hline $\mathrm{BMI}, \mathrm{kg} / \mathrm{m}^{2}$ & $24 \cdot 6 \pm 3 \cdot 3$ & $25 \cdot 0 \pm 3 \cdot 3$ & 0.047 \\
\hline WC, $\mathrm{cm}$ & $89.6 \pm 9.0$ & $91.0 \pm 9.0$ & 0.021 \\
\hline Exercise & & & 0.597 \\
\hline None & $212(45 \cdot 6)$ & $182(46.8)$ & \\
\hline 1 time/week & $32(6.9)$ & $30(7 \cdot 7)$ & \\
\hline 2 times/week & $44(9 \cdot 5)$ & $33(8.5)$ & \\
\hline 3 times/week & $53(11.4)$ & $37(9 \cdot 5)$ & \\
\hline 4 times/week & $23(4 \cdot 9)$ & $29(7 \cdot 5)$ & \\
\hline$\geq 5$ times/week & $101(21.7)$ & $78(20.1)$ & \\
\hline Exercise $\geq 3$ times/week & $177(38.1)$ & $144(37.0)$ & 0.808 \\
\hline Smoking & & & 0.405 \\
\hline Never & $285(61.3)$ & $225(57.8)$ & \\
\hline Former & $139(29.9)$ & $120(30.8)$ & \\
\hline Current & $41(8.8)$ & $44(11.3)$ & \\
\hline Alcohol & & & 0.004 \\
\hline Never & $119(25 \cdot 6)$ & $100(25 \cdot 7)$ & \\
\hline Former & $245(52.7)$ & $168(43.2)$ & \\
\hline Current & $101(21.7)$ & $121(31.1)$ & \\
\hline \multicolumn{4}{|l|}{ Comorbidities } \\
\hline Hypertension & $349(75.1)$ & $296(76.1)$ & 0.786 \\
\hline Diabetes & $121(26.0)$ & $115(29.6)$ & 0.282 \\
\hline Dyslipidemia & $119(25 \cdot 6)$ & $114(29 \cdot 3)$ & 0.256 \\
\hline Stroke & $26(5 \cdot 6)$ & $45(11.6)$ & 0.002 \\
\hline Myocardial infarction & $62(13 \cdot 3)$ & $45(11.6)$ & 0.501 \\
\hline Heart failure $^{\mathrm{a}}$ & $40(8.6)$ & $30(7 \cdot 7)$ & 0.729 \\
\hline Malignancy & $23(4.9)$ & $19(4.9)$ & 1.000 \\
\hline \multicolumn{4}{|l|}{ Family history } \\
\hline Hypertension & $208(44.7)$ & $181(46.5)$ & 0.648 \\
\hline Diabetes & $94(20.2)$ & $79(20.3)$ & 1.000 \\
\hline CV death & $15(3.2)$ & $13(3 \cdot 3)$ & 1.000 \\
\hline \multicolumn{4}{|l|}{ Medications } \\
\hline Antiplatelet agent ${ }^{\mathrm{b}}$ & $292(63.2)$ & $233(59.9)$ & 0.323 \\
\hline Statin & $224(48.2)$ & $169(43.4)$ & 0.190 \\
\hline ACEI & $41(8.8)$ & $39(10.0)$ & 0.627 \\
\hline ARB & $289(62.2)$ & $209(53.7)$ & 0.016 \\
\hline $\mathrm{CCB}$ & $226(48.6)$ & $197(50.6)$ & 0.599 \\
\hline Beta-blocker & $208(44.7)$ & $149(38.3)$ & 0.068 \\
\hline Diuretics & $90(19.4)$ & $63(16.2)$ & 0.267 \\
\hline
\end{tabular}


Table 1. Continued

\begin{tabular}{|c|c|c|c|}
\hline Characteristic & $\begin{array}{l}\text { Controlled HTN } \\
\qquad(\mathrm{n}=465)\end{array}$ & $\begin{array}{l}\text { Masked uncontrolled HTN } \\
\qquad(\mathrm{n}=389)\end{array}$ & $p$ value \\
\hline Other antihypertensive & $47(10.1)$ & $30(7 \cdot 7)$ & 0.273 \\
\hline No. of antihypertensive drugs & & & 0.009 \\
\hline 1 & $172(37.0)$ & $186(47.8)$ & \\
\hline 2 & $180(38.7)$ & $133(34.2)$ & \\
\hline$\geq 3$ & $113(24 \cdot 3)$ & $70(18.0)$ & \\
\hline \multicolumn{4}{|l|}{ Office BP } \\
\hline Office SBP, mmHg & $119.7 \pm 9.7$ & $129 \cdot 3 \pm 8.8$ & $<0.001$ \\
\hline Office DBP, mmHg & $73.6 \pm 7.6$ & $78.7 \pm 7 \cdot 3$ & $<0.001$ \\
\hline Heart rate, beats/min & $71.5 \pm 11.1$ & $72.7 \pm 10.4$ & 0.096 \\
\hline \multicolumn{4}{|l|}{ Ambulatory blood pressure monitoring } \\
\hline 24-hour mean SBP, mmHg & $118.0 \pm 7.0$ & $134.9 \pm 8.8$ & $<0.001$ \\
\hline 24-hour mean DBP, mmHg & $72.2 \pm 5.1$ & $82.1 \pm 6.8$ & $<0.001$ \\
\hline Daytime mean SBP, mmHg & $121.0 \pm 8.0$ & $137.6 \pm 9.0$ & $<0.001$ \\
\hline Daytime mean DBP, mmHg & $74 \cdot 3 \pm 5 \cdot 7$ & $84.1 \pm 7 \cdot 3$ & $<0.001$ \\
\hline Nighttime mean SBP, mmHg & $109.6 \pm 10.5$ & $126.6 \pm 13.7$ & $<0.001$ \\
\hline Nighttime mean DBP, mmHg & $66.3 \pm 6.9$ & $76.2 \pm 8.7$ & $<0.001$ \\
\hline \multicolumn{4}{|l|}{ Echocardiography } \\
\hline Ejection fraction & $59.1 \pm 7.1$ & $58.8 \pm 6.8$ & 0.514 \\
\hline LVMI (BSA), g/m² & $96.5 \pm 25.0$ & $102.3 \pm 23.2$ & 0.001 \\
\hline \multicolumn{4}{|l|}{ Laboratory tests } \\
\hline Hemoglobin, g/dL & $13.4 \pm 1.7$ & $13.5 \pm 1.8$ & 0.384 \\
\hline Triglyceride, mg/dL & $119(87-152)$ & $118(85-159)$ & 0.983 \\
\hline Total cholesterol, mg/dL & $167.6 \pm 40.8$ & $173 \cdot 3 \pm 40.7$ & 0.041 \\
\hline HDL-C, mg/dL & $46.0 \pm 11.0$ & $46.0 \pm 11.8$ & 0.938 \\
\hline $\mathrm{LDL}-\mathrm{C}, \mathrm{mg} / \mathrm{dL}$ & $96.6 \pm 35 \cdot 3$ & $100.7 \pm 32.7$ & 0.085 \\
\hline eGFR (CKD-EPI), mL/min/1.73 $\mathrm{m}^{2}$ & $81.9 \pm 20.5$ & $83.3 \pm 21.0$ & 0.316 \\
\hline Fasting glucose, mg/dL & $110.6 \pm 26.9$ & $109.9 \pm 27.5$ & 0.703 \\
\hline HgAic, \% & $6.1 \pm 0.9$ & $6.1 \pm 1.0$ & 0.499 \\
\hline
\end{tabular}

Values are presented as number (\%), mean \pm standard deviation, or median (interquartile range).

HTN, hypertension; MUCH, masked uncontrolled hypertension; BMI, body mass index; WC, waist circumference; CV, cardiovascular; ACEI, angiotensin-converting enzyme inhibitor; ARB, angiotensin II receptor blocker; CCB, calcium channel blocker; BP, blood pressure; SBP, systolic blood pressure; DBP, diastolic blood pressure; LVMI, left ventricular mass index; BSA, body surface area; HDL-C, high-density lipoprotein cholesterol; LDL-C, low-density lipoprotein cholesterol; eGFR, estimated glomerular filtration rate; CKD-EPI, Chronic Kidney Disease Epidemiology Collaboration; HgA1c, hemoglobin A1c.

${ }^{a}$ Heart failure was defined as the composite of hypertrophic cardiomyopathy, dilated cardiomyopathy, moderate to severe valve dysfunction, and ejection fraction $<40 \%$.

${ }^{\mathrm{b}}$ Antiplatelet agents including aspirin, clopidogrel, and a new P2Y12 inhibitor.

of antihypertensive drugs and the presence of $\mathrm{MUCH}$, using logistic regression models among patients taking a single antihypertensive drug (Supplementary Table 1).
There was no significant difference in the occurrence of MUCH among the five antihypertensive drug groups (angiotensin-converting enzyme inhibitors, angiotensin 


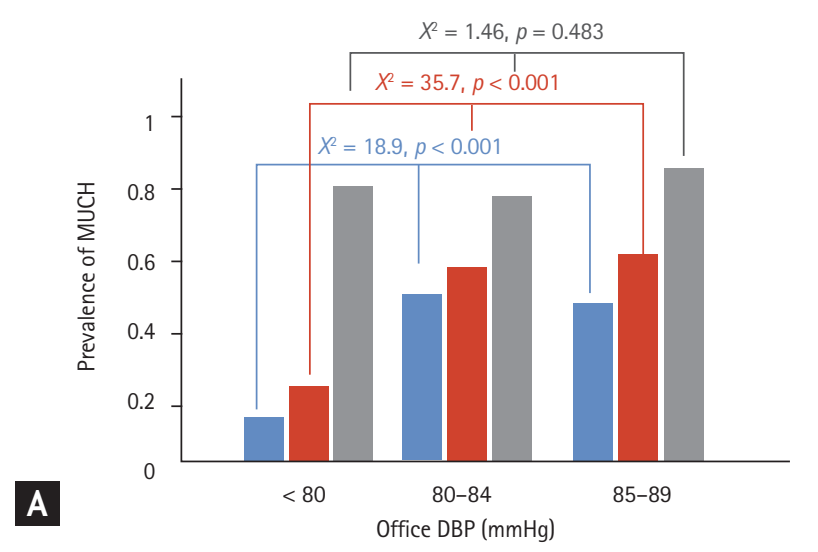

Office SBP $\quad \square<120 \mathrm{mmHg}$

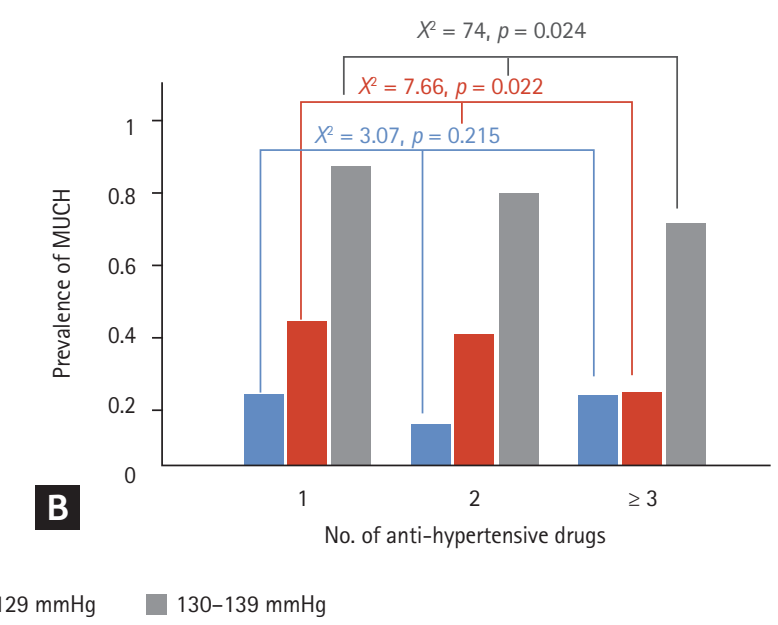

Figure 2. Prevalence of masked uncontrolled hypertension (MUCH) according to blood pressure levels. (A) MUCH was more frequent in patients with high systolic blood pressure (SBP) and diastolic blood pressure (DBP). However, when office SBP was $\geq 130 \mathrm{mmHg}$, the frequencies of MUCH were comparably high. (B) The frequencies of MUCH did not differ according to the number of antihypertensive drugs; however, when the SBP was $\geq 130 \mathrm{mmHg}$, the frequencies of MUCH decreased with increasing numbers of antihypertensive drugs.

II receptor blockers, calcium channel blockers, diuretics, and beta-blockers). The first multivariate model, including office SBP, office DBP, stroke, LVH, dyslipidemia, and $H R \geq 75$ beats/minute as covariates (multivariate model 1), showed that no antihypertensive drugs were associated with the occurrence of MUCH. In addition, multivariate model 2, which excluded variables that could influence drug choice, also showed that there was no difference among antihypertensive drugs.

\section{Prediction model for $\mathrm{MUCH}$}

Using the predictors included in the best-fit model derived from the multivariate logistic regression analysis, we developed a scoring system to predict $\mathrm{MUCH}$ in patients with apparently controlled office BP (Fig. 3 and Supplementary Table 2). The maximum point was set to 10 with an office SBP of 130 to $139 \mathrm{mmHg}$ so that the scoring system could be more easily comprehended and utilized. Subsequently, the total score ranged from o to 26 . In the ROC curve analysis, the scoring system showed good diagnostic performance for $\mathrm{MUCH}$, yielding a C-index of 0.839 (95\% CI, 0.813 to 0.866 ), sensitivity of 0.792 , specificity of 0.776 , PPV of 0.748 , and NPV of 0.817 at a cut-off value of 9.6 points (Fig. 4A). The observed frequencies of MUCH increased gradually with the total score and were in close agreement with the probabilities predicted using the scoring system (Hosmer-Lemeshow goodness-of-fit test, $p=0.999$ ) (Fig. $4 \mathrm{~B}$ and Supplementary Table 3). An internal validation test using 1000 bootstrap samples showed that the C-index of the scoring system had only a small optimism value (o.020), and calibration between the observed and predicted probability of MUCH estimated using the same bootstrap resamples produced values of 0.031 for the mean absolute error and 0.062 for the o.9th quantile absolute error (Supplementary Fig. 2).

\section{DISCUSSION}

In this study, we found that more than half of the patients on antihypertensive drugs who appeared to have controlled office BP actually had MUCH. Suboptimal office SBP and DBP levels, as well as underuse of antihypertensive drugs, increased HR, dyslipidemia, previous stroke, and LVH were strongly associated with an increased risk of $\mathrm{MUCH}$. The proposed model employing these clinical predictors showed high accuracy for predicting the presence of $\mathrm{MUCH}$ in an outpatient clinic setting.

Guidelines for the management of hypertension $[13,17,18]$ emphasize the importance of 24 -hour ABPM, 
Table 2. Prevalence of MUCH in various clinical settings

\begin{tabular}{|c|c|c|c|c|}
\hline Variable & No. & MUCH & $\%(95 \% \mathrm{CI})$ & OR $(95 \% \mathrm{CI}), p$ value \\
\hline Total & 854 & 389 & $44.1(40.8-47.5)$ & \\
\hline \multicolumn{5}{|l|}{ Office SBP } \\
\hline$<120 \mathrm{mmHg}$ & 251 & 41 & $16.3(11.8-20.9)$ & Reference $^{\mathrm{b}}$ \\
\hline $120-129 \mathrm{mmHg}$ & 286 & 100 & $35.0(29.4-40.5)$ & $2.75(1.82-4.16),<0.001$ \\
\hline $130-139 \mathrm{mmHg}$ & 317 & 248 & $78.2(73.7-82.8)$ & $18.4(12.0-28.2),<0.001$ \\
\hline \multicolumn{5}{|l|}{ Office DBP } \\
\hline$<80 \mathrm{mmHg}$ & 536 & 174 & $32.5(28.5-36.4)$ & Reference \\
\hline $80-84 \mathrm{mmHg}$ & 203 & 132 & $65.0(58.5-71.6)$ & $3.87(2.75-5.44),<0.001$ \\
\hline $85-89 \mathrm{mmHg}$ & 115 & 83 & $72.2(64.0-80.4)$ & $5.40(3.45-8.43),<0.001$ \\
\hline \multicolumn{5}{|c|}{ No. of antihypertensive drugs } \\
\hline$\geq 3$ & 183 & 70 & $38.3(31.2-45 \cdot 3)$ & Reference \\
\hline 2 & 313 & 133 & $42.5(37.0-48.0)$ & $1.29(0.88-1.88), 0.183$ \\
\hline 1 & 358 & 186 & $52.0(46.8-57.1)$ & $1.71(1.71-2.48), 0.005$ \\
\hline \multicolumn{5}{|l|}{ History of stroke } \\
\hline No & 783 & 344 & $43.9(40.5-47.4)$ & Reference \\
\hline Yes & 71 & 45 & $63.4(52.2-74.6)$ & $2.21(1.34-3.65), 0.002$ \\
\hline \multicolumn{5}{|l|}{ Dyslipidemia } \\
\hline No & 621 & 275 & $44.3(40.4-48.2)$ & Reference \\
\hline Yes & 233 & 114 & $48.9(42.5-55 \cdot 3)$ & $1.21(0.89-1.63), 0.225$ \\
\hline \multicolumn{5}{|l|}{$\mathrm{HR} \geq 75$ beats $/ \mathrm{min}^{\mathrm{a}}$} \\
\hline No & 369 & 154 & $41.7(36.7-46.8)$ & Reference \\
\hline Yes & 486 & 235 & $48.5(44.0-52.9)$ & $1.41(1.06-1.86), 0.017$ \\
\hline \multicolumn{5}{|l|}{$\mathrm{BMI} \geq 25 \mathrm{~kg} / \mathrm{m}^{2}$} \\
\hline No & 475 & 201 & $42.3(37.9-46.8)$ & Reference \\
\hline Yes & 379 & 188 & $49 \cdot 6(44.6-54.6)$ & $1.34(1.02-1.76), 0.034$ \\
\hline \multicolumn{5}{|c|}{$W C \geq 90 \mathrm{~cm}$ for men, $\geq 85 \mathrm{~cm}$ for women } \\
\hline No & 329 & 135 & $41.0(35.7-46.3)$ & Reference \\
\hline Yes & 525 & 254 & $48.4(44.1-52.7)$ & $1.35(1.02-1.78), 0.036$ \\
\hline \multicolumn{5}{|l|}{$\mathrm{LVH}^{\mathrm{a}}$} \\
\hline No & 569 & 240 & $42.2(38.1-46.2)$ & Reference \\
\hline Yes & 285 & 149 & $52.3(46.5-58.1)$ & $1.50(1.13-2.00), 0.005$ \\
\hline \multicolumn{5}{|c|}{ Current alcohol intake } \\
\hline No & 632 & 268 & $42.4(38.6-46.3)$ & Reference \\
\hline Yes & 222 & 121 & $54.5(48.0-61.1)$ & $1.63(1.20-2.21), 0.002$ \\
\hline \multicolumn{5}{|l|}{ Antiplatelet agent } \\
\hline No & 405 & 182 & $44.9(40.1-49.8)$ & Reference \\
\hline Yes & 449 & 207 & $46.1(41.5-50.7)$ & $1.05(0.80-1.37), 0.733$ \\
\hline \multicolumn{5}{|l|}{ Current smoking } \\
\hline No & 769 & 345 & $44.9(41.3-48.4)$ & Reference \\
\hline Yes & 85 & 44 & $51.8(41.1-62.4)$ & $1.32(0.84-2.07), 0.227$ \\
\hline \multicolumn{5}{|l|}{ Sex } \\
\hline Female & 377 & 153 & $40.6(35.6-45 \cdot 5)$ & Reference \\
\hline Male & 477 & 236 & $49 \cdot 5(45 \cdot 0-54.0)$ & $1.43(1.09-1.88), 0.010$ \\
\hline \multicolumn{5}{|l|}{ Age $\geq 50$ years ${ }^{\mathrm{a}}$} \\
\hline No & 141 & 70 & $49.6(41.4-57.9)$ & Reference \\
\hline Yes & 713 & 319 & $44.7(41.1-48.4)$ & $0.82(0.57-1.18), 0.286$ \\
\hline \multicolumn{5}{|l|}{ Diabetes } \\
\hline No & 618 & 274 & $44.3(40.4-48.3)$ & Reference \\
\hline Yes & 236 & 115 & $48.7(42.4-55.1)$ & $1.19(0.88-1.61), 0.249$ \\
\hline
\end{tabular}

MUCH, masked uncontrolled hypertension; CI, confidence interval; OR, odds ratio; SBP, systolic blood pressure; DBP, diastolic blood pressure; HR, heart rate; BMI, body mass index; WC, waist circumference; LVH, left ventricular hypertrophy.

${ }^{a}$ Cut-off values of the continuous variables were determined using univariate logistic regression models with a restrictive cubic spline fit for the variables.

${ }^{\mathrm{b}}$ Categories used as references. 
Table 3. Multivariate logistic regression model of the predictors for masked hypertension

\begin{tabular}{|c|c|c|c|}
\hline Variable & $\mathrm{OR}^{\mathrm{a}}$ & $95 \% \mathrm{CI}$ & $p$ value \\
\hline \multicolumn{4}{|l|}{ Office SBP } \\
\hline $120-129 \mathrm{mmHg}$ vs. $<120 \mathrm{mmHg}$ & 2.22 & $1.42-3.46$ & $<0.001$ \\
\hline 130-139 mmHg vs. $<120 \mathrm{mmHg}$ & 14.9 & $9.34-23.7$ & $<0.001$ \\
\hline \multicolumn{4}{|l|}{ Office DBP } \\
\hline $80-84 \mathrm{mmHg}$ vs. $<80 \mathrm{mmHg}$ & 2.47 & $1.64-3.71$ & $<0.001$ \\
\hline $85-89 \mathrm{mmHg}$ vs. $<80 \mathrm{mmHg}$ & 3.15 & $1.87-5.30$ & $<0.001$ \\
\hline Stroke & 2.61 & $1.39-4.89$ & 0.003 \\
\hline $\mathrm{LVH}$ & 1.96 & $1.36-2.85$ & $<0.001$ \\
\hline \multicolumn{4}{|l|}{ No. of antihypertensive drugs } \\
\hline 1 vs. $\geq 3$ & 2.39 & $1.52-3.77$ & $<0.001$ \\
\hline 2 vs. $\geq 3$ & 1.58 & $1.00-2.51$ & 0.048 \\
\hline Dyslipidemia & 1.66 & $1.14-2.41$ & 0.009 \\
\hline Heart rate $\geq 75$ beats $/ \mathrm{min}$ & 1.45 & $1.02-2.06$ & 0.040 \\
\hline
\end{tabular}

The multivariate model was reduced using a backward variable selection process (cut-off criteria, $p<0.05$ ).

OR, odds ratio; CI, confidence interval; SBP, systolic blood pressure; DBP, diastolic blood pressure; LVH, left ventricular hypertrophy.

${ }^{a}$ Covariates include age, sex, body mass index, waist circumference, exercise < 3 times/week, current alcohol intake or smoking, diabetes and dyslipidemia, past histories of stroke, myocardial infarction, and malignancies; family histories of hypertension, diabetes, and cardiovascular death; types and number of antihypertensive medications, left ventricular mass index, medications, office SBP, office DBP, heart rate, and low-density lipoprotein cholesterol levels.

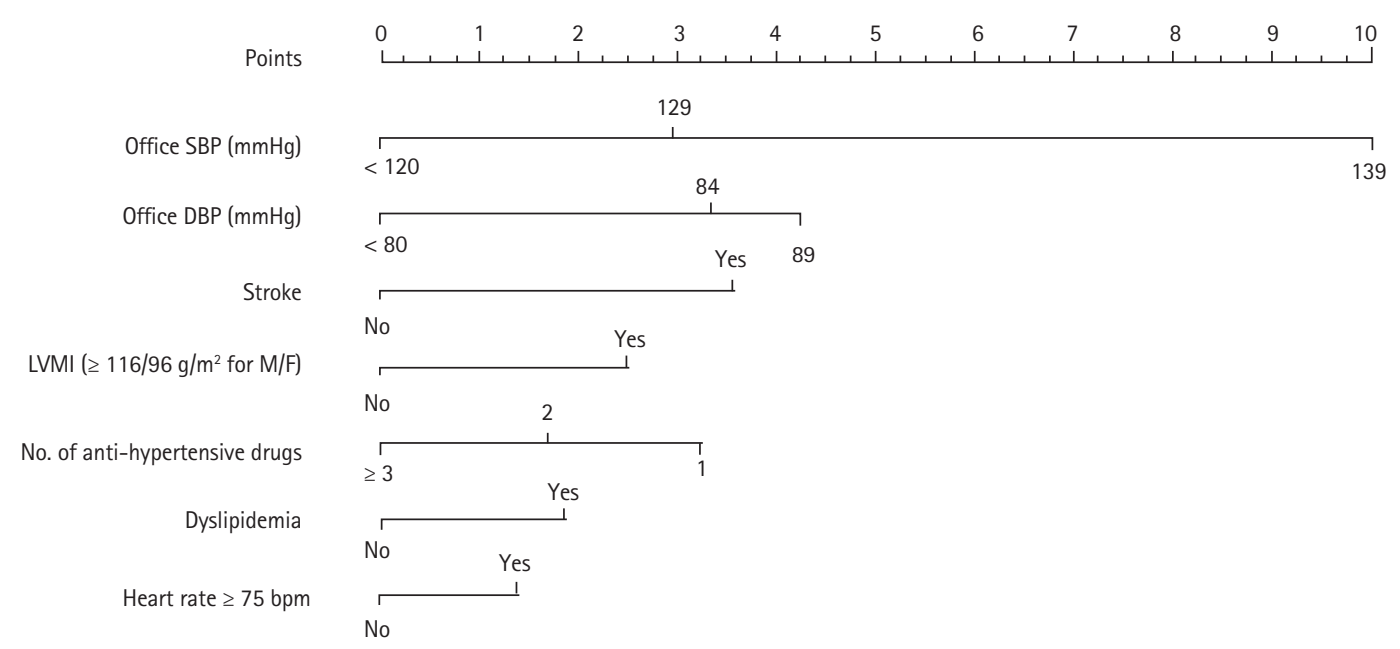

Figure 3. Nomogram of the scoring system predicting for masked uncontrolled hypertension. SBP, systolic blood pressure; DBP, diastolic blood pressure; LVMI, left ventricular mass index.

making it important for the diagnosis of white-coat hypertension and $\mathrm{MH} . \mathrm{MUCH}$, which is defined by normal office BP and elevated out-of-office BP in patients receiving treatment for hypertension $[2,9]$, can also be detected using 24-hour ABPM. Recent studies using me- ta-analyses, including prospective cohort studies, have shown that patients with MUCH face increased risks of cardiovascular events and all-cause death (hazard ratio, 1.80; $95 \%$ CI, 1.57 to 2.06) compared with patients with controlled hypertension [9]. In addition, because of the 

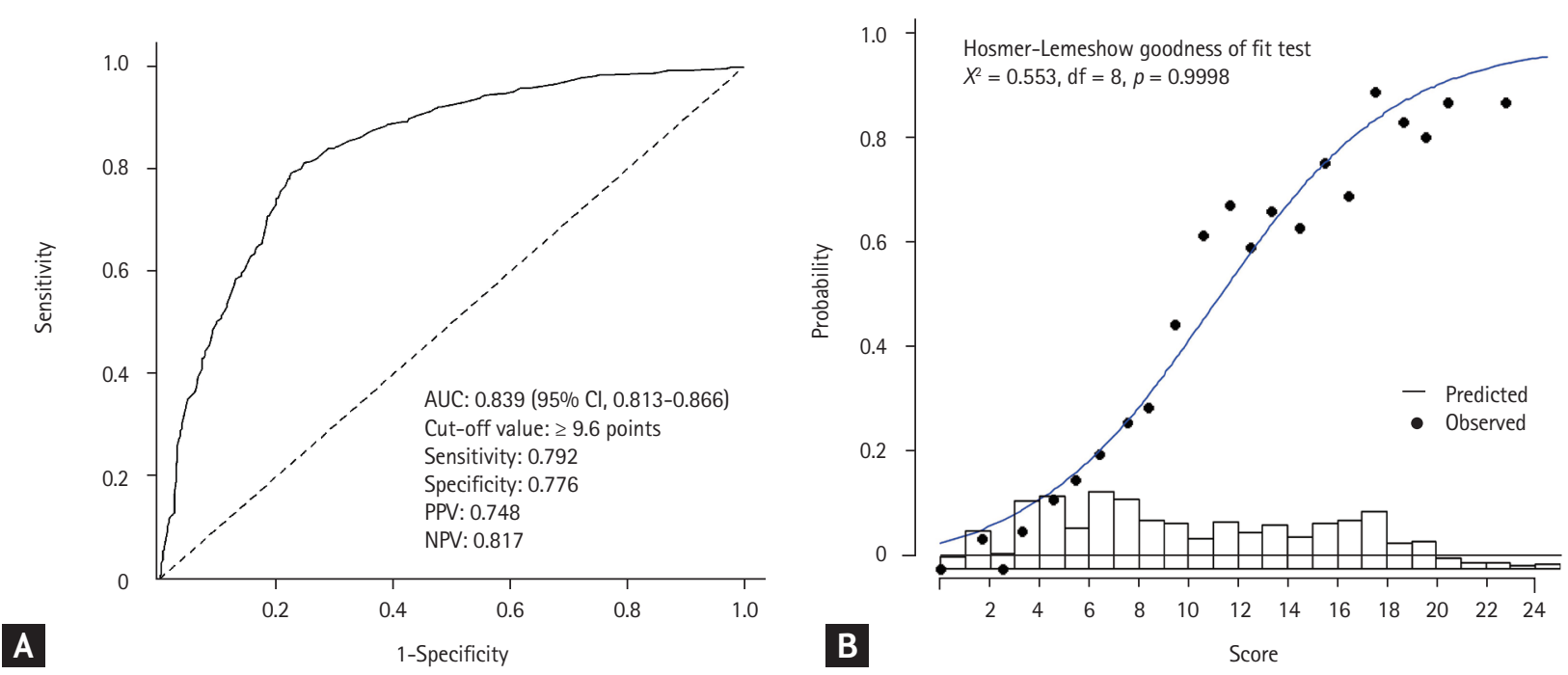

Figure 4. Diagnostic performances and the goodness of fit of the score system predicting. (A) Receiver operating characteristic curve analysis showed that the score system showed good diagnostic performance for masked uncontrolled hypertension. (B) Hosmer-Lemeshow goodness-of-fit test. AUC, area under the curve; CI, confidence interval; PPV, positive predictive value; $\mathrm{NPV}$, negative predictive value.

high incidence of cardiovascular events and predictable cardiac events in $\mathrm{MH}$ [19], it is important to know its clinical features and predictors in patients receiving hypertension treatment, especially in the MUCH subtype.

Our results underline the importance of the so-called high-normal office BP, which can be defined as an office SBP of 130 to $139 \mathrm{mmHg}$ or DBP of 85 to $89 \mathrm{mmHg}$. The prevalence of MUCH increased 15-fold and 3-fold in patients with high-normal office SBP and DBP, respectively. A previous study using the Spanish Society of Hypertension ABPM registry data reported the prevalence of MUCH as 31.1\% among patients with treated and controlled office $\mathrm{BP}(<140 / 90 \mathrm{mmHg})[1]$. The researchers in the Spanish registry study showed that the presence of MUCH was also associated with high-normal office SBP and DBP, similar to our results.

Whether high-risk characteristics correlate with MUCH remains controversial. However, several studies have indicated that the risk profile of $\mathrm{MH}$ patients is in line with the risk profile of patients with MUCH. Metabolic disorders, including central obesity, hypertension, diabetes, and dyslipidemia, also increase the risk of $\mathrm{MH}$ [20-22]. Our results confirmed that patients with MUCH have more metabolic disturbances than patients with controlled hypertension. BMI $\geq 25 \mathrm{~kg} / \mathrm{m}^{2}$ and WC were significantly associated with $\mathrm{MUCH}$, and dyslipid- emia was an independent relevant factor for $\mathrm{MUCH}$ in our study. In addition, a history of stroke was an independent risk factor for MUCH. MH has been associated with a higher risk of stroke [5], but the mechanism by which a history of stroke increases the risk of $\mathrm{MH}$ has yet to be determined. Moreover, one of the markers of target organ damage, $\mathrm{LVH}$, was associated with $\mathrm{MUCH}$ in our study. Therefore, it seems reasonable to perform ABPM in individuals with these metabolically high-risk characteristics or target organ damage, such as LVH.

Previous studies have demonstrated that patients with excessive alcohol intake have an increased incidence of $\mathrm{MH}[23,24]$. Ishikawa et al. [23] reported that regular alcohol consumption increased the risk of MUCH by 1.76fold in patients with stable hypertension. Our study supports previous findings that patients with MUCH were significantly more likely to be alcohol drinkers, with a 1.63-fold increased risk of MUCH compared to patients with controlled hypertension on univariate logistic regression analyses. Although current alcohol intake was not an independent risk factor for $\mathrm{MUCH}$, in patients with high-normal BP or other metabolic risks, alcohol consumption may be considered and assessed as a factor affecting MUCH, regardless of the controlled-BP status.

In this study, we proposed a prediction model for MUCH that appeared to provide high diagnostic ac- 
curacy and goodness of fit for clinicians to identify patients with $\mathrm{MUCH}$ in outpatient settings using several easily available clinical features. The internal validation and calibration results obtained using the bootstrap resampling technique also showed sufficient reliability and validity of the model. It may be difficult for clinicians to notice the presence of MUCH in regular outpatients on antihypertensive drugs, since the condition is asymptomatic and, to date, office BP remains the main BP measurement tool and lacks any widely known clinical clues suggestive of MUCH. As MUCH is highly prevalent in patients with apparently controlled BP and is known to result in worse clinical outcomes [9], clinicians should be mindful of identifying MUCH in those patients with the predictors for MUCH. Although sufficient external validations are required, the proposed prediction model may be useful in providing clinical cues for clinicians to prescribe ABPM in regular outpatients on antihypertensive drugs who satisfy some of the predictors.

Furthermore, the strong association between high-normal office BP and the lower number of antihypertensive drugs in the prediction model could provide the valuable insight that suboptimal office BP control through the underuse of antihypertensive drugs plays an important role in the development of MUCH. The significant interaction between the number of antihypertensive drugs and office SBP on the lower risk of MUCH may also underscore the role of suboptimal antihypertensive therapy in the development of MUCH. Although sub-analysis was performed according to the type of single antihypertensive drug used to evaluate whether the risk of $\mathrm{MUCH}$ varied with the drug of choice, there was no significant difference. Further study will be needed in the future to determine which combination of antihypertensive drugs affects the risk of MUCH. In addition to suboptimal office BP, studies to determine MUCH-specific parameters to filter out those patients with actual uncontrolled BP will be needed.

This study has several limitations. First, as with any cross-sectional study, the associations between the variables observed could not establish a causal relationship. Second, we did not assess medication non-compliance, a well-known cause of uncontrolled BP in patients on antihypertensive drugs. However, a recent study showed that unlike uncontrolled hypertension, $\mathrm{MUCH}$ is not attributable to medication non-adherence [25]. Third, the duration of history of comorbidities, including hypertension and stroke, may be an important factor in uncontrolled hypertension, but data on these were insufficient. Approximately $8 \%$ of the original data were missing, with the rate of missing values varying from ०\% to $58 \%$ among the variables. We did not exclude records with missing values and performed multiple imputations to prevent a loss of statistical power due to exclusion of data [26]. Although multiple imputation using a bootstrap expectation-maximization algorithm is a frequently employed method for imputations, imputed data may have had unanticipated effects on the results. Finally, we validated and calibrated the prediction model internally using bootstrap resampling. Although resampling is a well-established method for internal validation, external validation using an independent dataset derived from a similar clinical settings will provide more confidence in the model.

MUCH is highly prevalent in patients with apparently controlled office BP. A borderline increase in office SBP and DBP, and underuse of antihypertensive drugs, as well as dyslipidemia, increased HRs, previous stroke, and LVH are independently associated with the presence of MUCH, which underscores the role of suboptimal treatment of hypertension in the development of MUCH. Clinicians should be mindful of the presence of MUCH in patients with these predictors, and the proposed prediction model may serve as a useful screening tool for MUCH to provide a clinical cue to prescribe 24hour ABPM to patients.

\section{KEY MESSAGE}

1. Among patients with treated hypertension and normal office blood pressure (BP), 45.6\% had masked uncontrolled hypertension (MUCH), which could be mistaken for good control by masking.

2. The borderline ranges of office systolic BP and diastolic BP were independently associated with a significantly increased risk of MUCH.

3. In this study, the proposed predictive model can act as a quick screening tool for MUCH before patients can perform 24-hour ambulatory BP monitoring. 


\section{Conflict of interest}

No potential conflict of interest relevant to this article was reported.

\section{Acknowledgments}

We wish to thank the Working Group of Blood Pressure Monitoring in Korean Society of Hypertension for managing the registry data construction.

\section{REFERENCES}

1. Banegas JR, Ruilope LM, de la Sierra A, et al. High prevalence of masked uncontrolled hypertension in people with treated hypertension. Eur Heart J 2014;35:3304-3312.

2. Franklin SS, O'Brien E, Staessen JA. Masked hypertension: understanding its complexity. Eur Heart J 2017;38:1112-1118.

3. Franklin SS, O’Brien E, Thijs L, Asayama K, Staessen JA. Masked hypertension: a phenomenon of measurement. Hypertension 2015;65:16-20.

4. Tientcheu D, Ayers C, Das SR, et al. Target organ complications and cardiovascular events associated with masked hypertension and white-coat hypertension: analysis from the Dallas Heart Study. J Am Coll Cardiol 2015;66:21592169 .

5. Ohkubo T, Kikuya M, Metoki H, et al. Prognosis of "masked" hypertension and "white-coat" hypertension detected by 24-h ambulatory blood pressure monitoring 10-year follow-up from the Ohasama study. J Am Coll Cardiol 2005;46:508-515.

6. Wang C, Zhang J, Li Y, et al. Masked hypertension, rather than white-coat hypertension, has a prognostic role in patients with non-dialysis chronic kidney disease. Int J Cardiol 2017;230:33-39.

7. Bobrie G, Chatellier G, Genes N, et al. Cardiovascular prognosis of "masked hypertension" detected by blood pressure self-measurement in elderly treated hypertensive patients. JAMA 2004;291:1342-1349.

8. Stergiou GS, Asayama K, Thijs L, et al. Prognosis of whitecoat and masked hypertension: international database of home blood pressure in relation to cardiovascular outcome. Hypertension 2014;63:675-682.

9. Pierdomenico SD, Pierdomenico AM, Coccina F, et al. Prognostic value of masked uncontrolled hypertension. Hypertension 2018;72:862-869.
10. Shin J, Park SH, Kim JH, et al. Discordance between ambulatory versus clinic blood pressure according to global cardiovascular risk group. Korean J Intern Med 2015;30:610-619.

11. Parati G, Stergiou G, O’Brien E, et al. European Society of Hypertension practice guidelines for ambulatory blood pressure monitoring. J Hypertens 2014;32:1359-1366.

12. Kang IS, Pyun WB, Shin J, et al. Higher blood pressure variability in white coat hypertension: from the Korean Ambulatory Blood Pressure Monitoring Registry. Korean Circ J 2016;46:365-373.

13. Williams B, Mancia G, Spiering W, et al. 2018 ESC/ESH guidelines for the management of arterial hypertension. Eur Heart J 2018;39:3021-3104.

14. Koo TY, Jeong JC, Lee Y, et al. Pre-transplant evaluation of donor urinary biomarkers can predict reduced graft function after deceased donor kidney transplantation. Medicine (Baltimore) 2016;95:e3076.

15. Harrell FE jr. Regression Modeling Strategies. New York (NY): Springer, 2001.

16. Lee Y, Shin JH, Park HC, Kim SG, Choi SI. A prediction model for renal artery stenosis using carotid ultrasonography measurements in patients undergoing coronary angiography. BMC Nephrol 2014;15:60.

17. Whelton PK, Carey RM, Aronow WS, et al. 2017 ACC/AHA/ AAPA/ABC/ACPM/AGS/APhA/ASH/ASPC/NMA/PCNA guideline for the prevention, detection, evaluation, and management of high blood pressure in adults: a report of the American College of Cardiology/American Heart Association Task Force on clinical practice guidelines. Hypertension 2018;71:e13-e115.

18. Lee HY, Shin J, Kim GH, et al. 2018 Korean Society of Hypertension guidelines for the management of hypertension: part II-diagnosis and treatment of hypertension. Clin Hypertens 2019;25:20.

19. Pierdomenico SD, Cuccurullo F. Prognostic value of white-coat and masked hypertension diagnosed by ambulatory monitoring in initially untreated subjects: an updated meta analysis. Am J Hypertens 2011;24:52-58.

20. Hanninen MR, Niiranen TJ, Puukka PJ, Jula AM. Metabolic risk factors and masked hypertension in the general population: the Finn-Home study. J Hum Hypertens 2014;28:421-426.

21. Colantonio LD, Anstey DE, Carson AP, et al. Metabolic syndrome and masked hypertension among African Americans: the Jackson Heart Study. J Clin Hypertens 
(Greenwich) 2017;19:592-600.

22. Cuspidi C, Facchetti R, Bombelli M, et al. Risk of new-onset metabolic syndrome associated with white-coat and masked hypertension: data from a general population. J Hypertens 2018;36:1833-1839.

23. Ishikawa J, Kario K, Eguchi K, et al. Regular alcohol drinking is a determinant of masked morning hypertension detected by home blood pressure monitoring in medicated hypertensive patients with well-controlled clinic blood pressure: the Jichi Morning Hypertension Research (J-MORE) study. Hypertens Res 2006;29:679686.
24. Ohira T, Tanigawa T, Tabata M, et al. Effects of habitual alcohol intake on ambulatory blood pressure, heart rate, and its variability among Japanese men. Hypertension 2009;:53:13-19.

25. Siddiqui M, Judd EK, Dudenbostel T, et al. Masked uncontrolled hypertension is not attributable to medication nonadherence. Hypertension 2019;74:652-659.

26. Hayati Rezvan P, Lee KJ, Simpson JA. The rise of multiple imputation: a review of the reporting and implementation of the method in medical research. BMC Med Res Methodol 2015;15:30. 


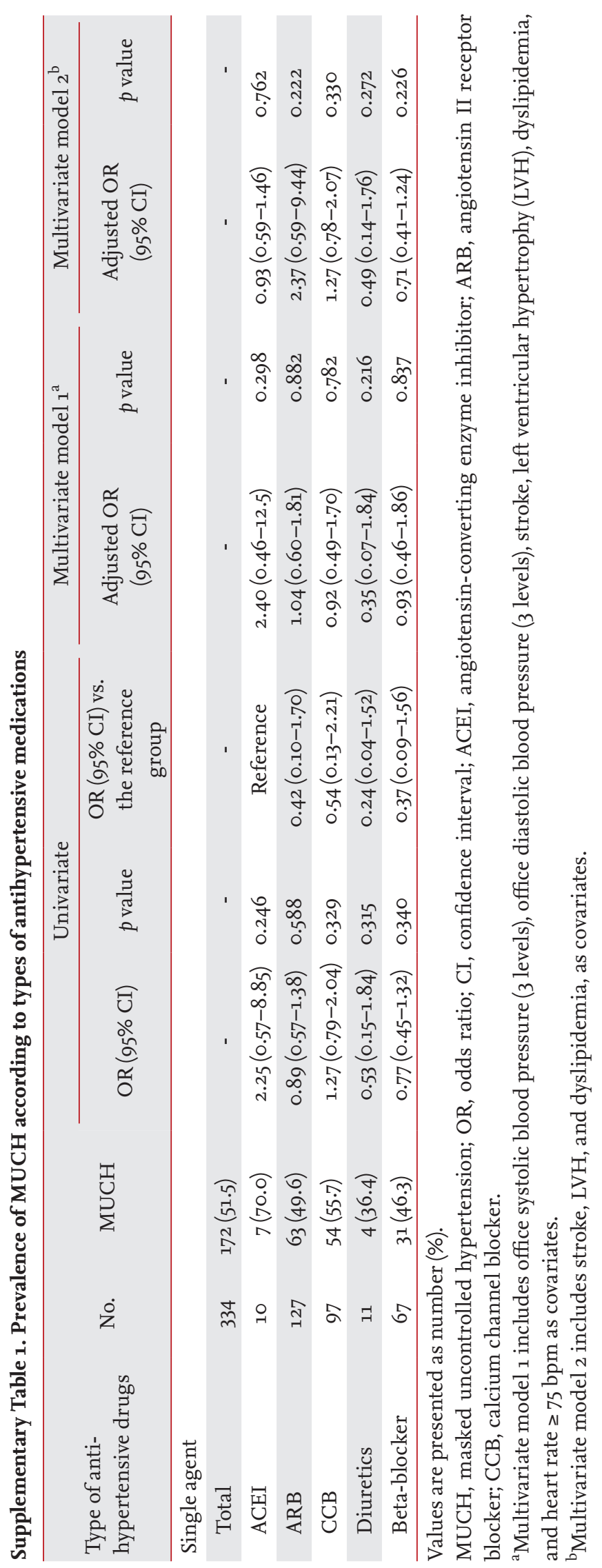


Supplementary Table 2. The score predicting masked hypertension

\begin{tabular}{|c|c|c|c|c|c|c|c|c|c|c|c|c|c|}
\hline \multicolumn{2}{|c|}{$\begin{array}{c}\text { Office SBP, } \\
\text { mmHg }\end{array}$} & \multicolumn{2}{|c|}{$\begin{array}{l}\text { Office DBP, } \\
\text { mmHg }\end{array}$} & \multicolumn{2}{|c|}{$\begin{array}{c}\text { History of } \\
\text { stroke }\end{array}$} & \multicolumn{2}{|c|}{$\begin{array}{c}\text { LVMI } \\
\geq 116 / 96 \mathrm{~g} / \mathrm{m}^{2} \\
\text { for male/female }\end{array}$} & \multicolumn{2}{|c|}{$\begin{array}{l}\text { No. of anti- } \\
\text { hypertensive } \\
\text { agents }\end{array}$} & \multicolumn{2}{|c|}{ Dyslipidemia } & \multicolumn{2}{|c|}{$\begin{array}{l}\text { Heart rate } \\
\geq 75 \mathrm{bpm}\end{array}$} \\
\hline Value & Scale & Value & Scale & Value & Scale & Value & Scale & Value & Scale & Value & Scale & Value & Scale \\
\hline$<120$ & 0 & $<80$ & 0 & No & 0 & No & 0 & $\geq 3$ & 0 & No & 0 & No & o \\
\hline $120-129$ & 3 & $80-84$ & $3 \cdot 3$ & Yes & 3.6 & Yes & 2.5 & 2 & 1.7 & Yes & 1.9 & Yes & 1.4 \\
\hline $130-139$ & 10 & $85-89$ & 4.2 & & & & & 1 & 3.2 & & & & \\
\hline
\end{tabular}

SBP, systolic blood pressure; DBP, diastolic blood pressure; LVMI, left ventricular mass index. 
Kim HJ, et al. Predictors of MUCH

Supplementary Table 3. Goodness of fit between observed probability and predicted probability

\begin{tabular}{|c|c|c|c|c|}
\hline Score & No. of patients & Observed frequency & Observed probability & Predicted probability \\
\hline $0.0-0.9$ & 11 & 0 & 0.000 & 0.048 \\
\hline $1.0-1.9$ & 36 & 2 & 0.056 & 0.074 \\
\hline $2.0-2.9$ & 8 & 0 & 0.000 & 0.091 \\
\hline $3.0-3.9$ & 70 & 5 & 0.071 & 0.110 \\
\hline $4.0-4.9$ & 61 & 8 & 0.131 & 0.147 \\
\hline $5.0-5.9$ & 47 & 8 & 0.170 & 0.181 \\
\hline $6.0-6.9$ & 73 & 16 & 0.219 & 0.222 \\
\hline $7.0-7.9$ & 54 & 15 & 0.278 & 0.280 \\
\hline $8.0-8.9$ & 52 & 16 & 0.308 & 0.330 \\
\hline $9.0-9.9$ & 43 & 20 & 0.465 & 0.398 \\
\hline $10.0-10.9$ & 33 & 21 & 0.636 & 0.473 \\
\hline $11.0-11.9$ & 39 & 27 & 0.692 & 0.544 \\
\hline $12.0-12.9$ & 36 & 22 & 0.611 & 0.601 \\
\hline $13.0-13.9$ & 44 & 30 & 0.682 & 0.654 \\
\hline $14.0-14.9$ & 20 & 13 & 0.650 & 0.720 \\
\hline $15.0-15.9$ & 53 & 41 & 0.774 & 0.773 \\
\hline $16.0-16.9$ & 45 & 32 & 0.711 & 0.815 \\
\hline $17.0-17.9$ & 54 & 49 & 0.907 & 0.855 \\
\hline $18.0-18.9$ & 20 & 17 & 0.850 & 0.888 \\
\hline $19.0-19.9$ & 28 & 23 & 0.821 & 0.910 \\
\hline $20.0-20.9$ & 9 & 8 & 0.889 & 0.928 \\
\hline $21.0-26.0$ & 18 & 16 & 0.889 & 0.961 \\
\hline
\end{tabular}




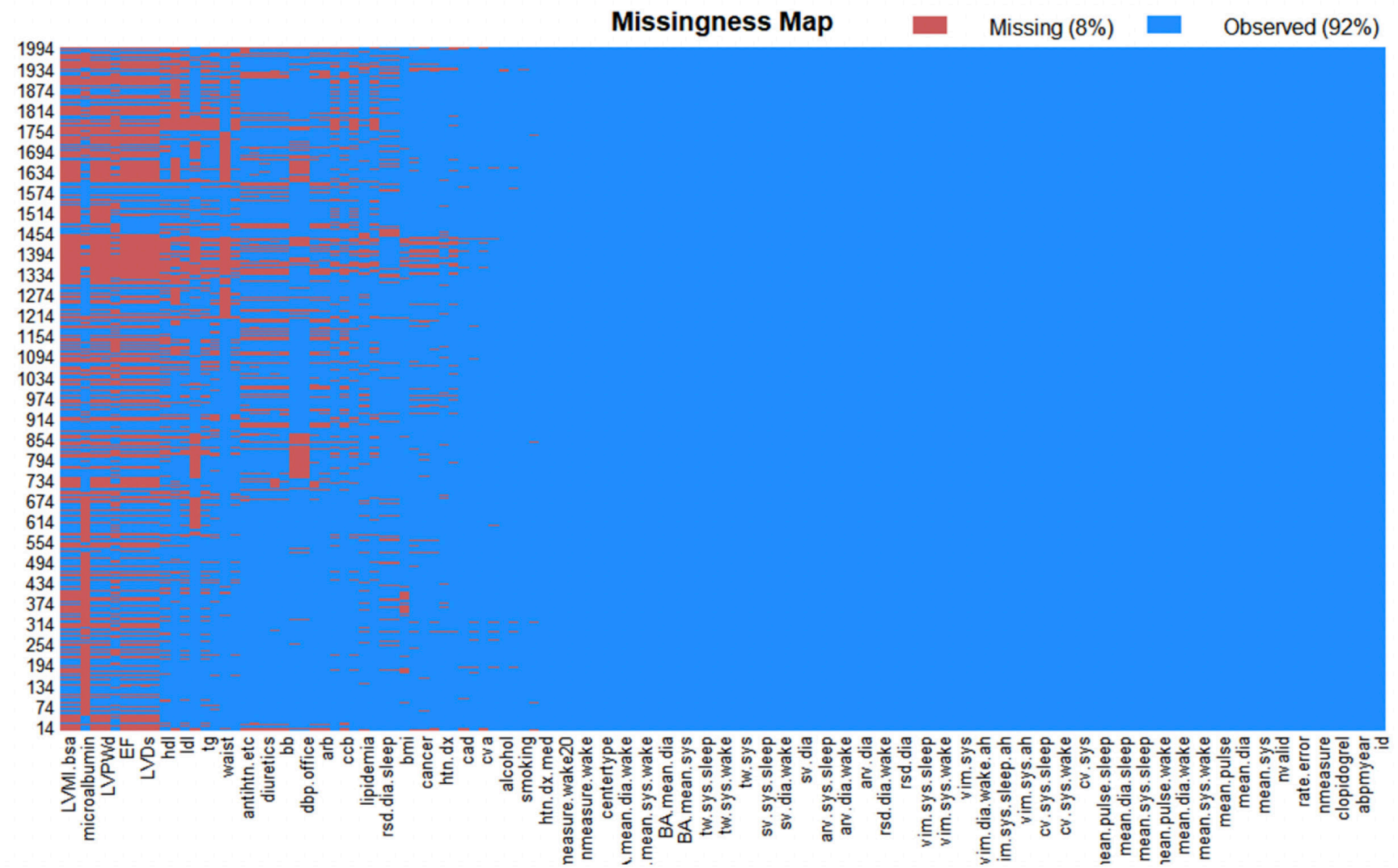

Supplementary Figure 1. Missing values in the dataset. There are $8 \%$ of missing values in the dataset. The rate of missing values vary between $0 \%$ and $58 \%$. Multiple imputation using an expectation and maximization algorithm was employed to fill up the missing values. 


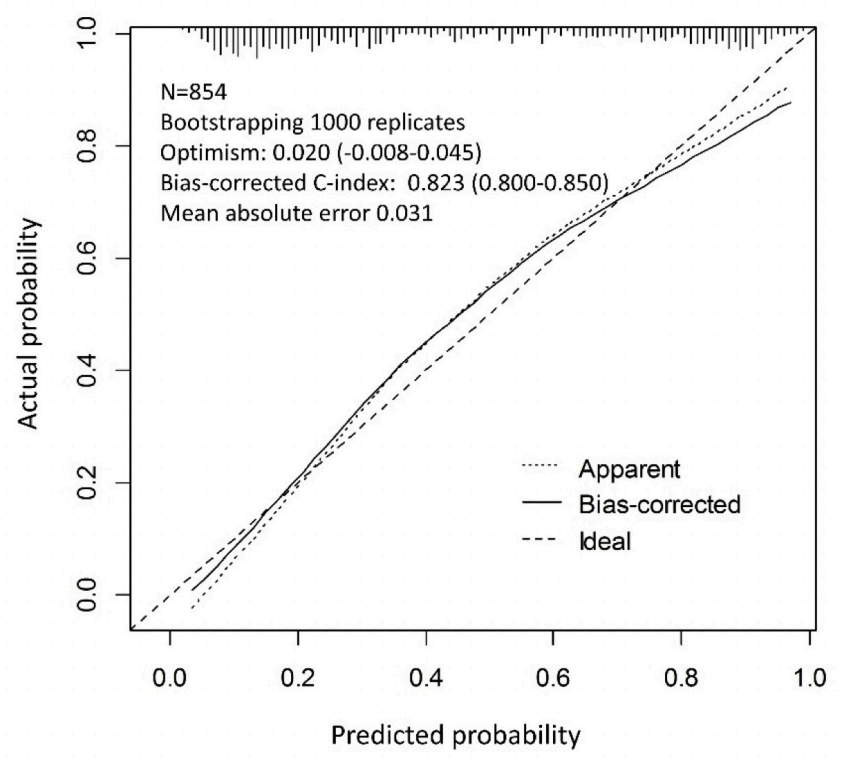

Supplementary Figure 2. Internal validation and calibration through bootstrap resampling. Internal validation using 1,000 bootstrap resamples showed that the optimism included in the C-index of the best model was o.o2o. Calibration using the 1,000 bootstrap resamples showed only a small amount of the mean absolute error (0.031) and the 0.9 quantile of absolute error (0.062). The bias between predicted probability and actual probability was generally larger in high-probability masked uncontrolled hypertension (MUCH) than in low-probability of MUCH. 